\title{
FORUM \\ Nach den gescheiterten Referenden: Was wird aus dem Außenminister der Union und dem Europäischen Auswärtigen Dienst?
}

\author{
Christoph Heusgen*
}

Seit dem In-Kraft-Treten des Vertrags von Amsterdam im Jahre 1999 hat die Gemeinsame Außen- und Sicherheitspolitik (GASP) der Europäischen Union eine beeindruckende Dynamik entfaltet.

Innerhalb kürzester Zeit gelang es, unter Führung des Hohen Repräsentanten für die GASP, Javier Solana, die institutionellen Voraussetzungen zu schaffen, die es der Union erlaubten, zu einem politischen Akteur zu werden. Hatten die Balkankriege in den Neunzigerjahren noch das Unvermögen der Europäer unter Beweis gestellt, auf ihrem eigenen Kontinent für Frieden und Stabilität zu sorgen, gelang es der Europäischen Union in der Folgezeit, ihrer Verantwortung gerecht zu werden und neu entstehende Krisen im Keim zu ersticken (beispielsweise im Jahre 2001 den Ausbruch eines neuen Kriegs in Mazedonien oder den drohenden Zerfall der Bundesrepublik Jugoslawien in 2002), einen wesentlichen Beitrag zum Krisenmanagement zu leisten (wie etwa in der Provinz Ituri im Kongo 2003 oder bei den innenpolitischen Auseinandersetzung in der Ukraine 2004), wichtige militärische Aufgaben zu übernehmen (beispielsweise in Bosnien die Ablösung von SFOR durch EUFOR im Jahr 2004) und als Vermittler bei regionalen Konflikten eine größere Rolle zu spielen (beispielsweise als Mitglied des Nahostquartetts, bei der Suche nach einer Lösung des Transnistrienkonflikts seit 2003).

Aufbauend auf den seit 1999 gemachten Erfahrungen sieht der Entwurf der Europäischen Verfassung eine Reihe von Bestimmungen vor, die die GASP noch wirksamer machen sollen. Nachdem es nun nicht danach aussieht, als ob wir in absehbarer Zeit damit rechnen können, dass die Verfassung in Kraft tritt, stellt sich die Frage, wie es weitergehen soll.

Dazu zunächst drei grundsätzliche Bemerkungen: Erstens können wir lange über Sinn und Unsinn von Referenden diskutieren. Aber Tatsache ist, dass die Volksbefragungen zur Europäischen Verfassung in Frankreich und den Niederlanden stattgefunden haben und dass sie negativ ausgegangen sind. Wir können deswegen jetzt nicht zur Tagesordnung übergehen, die Meinung der Bürger ignorieren und die Verfassung implementieren, als ob nichts geschehen wäre.

Zweitens wissen wir aber auch um die Ergebnisse von Meinungsumfragen, in denen die Bürger in allen Mitgliedstaaten der Europäischen Union - einschließlich Frankreichs, der Niederlande und Großbritanniens - auf die Frage, in welchen Politikbereichen sie eine stärkere europäische Integration wünschen, regelmäßig die Außen- und Sicherheitspolitik an erster Stelle nennen.

Drittens hat bis heute niemand ernsthaft der vom Europäischen Rat im Dezember 2003 angenommenen Europäischen Sicherheitsstrategie widersprochen, die hervorhebt, dass die europäischen Nationalstaaten im Zeitalter der Globalisierung allein nicht in der Lage sind, mit

* Dr. Christoph Heusgen, Leiter Politischer Stab, Generalsekretaritat des Rates der Europäischen Union. Der Inhalt dieses Beitrags gibt die persönliche Meinung des Autors wieder. 
den Herausforderungen fertig zu werden, denen sie gegenüberstehen: der Verbreitung von Massenvernichtungswaffen, dem Terrorismus, regionalen Krisen, dem Organisierten Verbrechen und dem Phänomen der , scheiternden Staaten'. Es geht nur im europäischen Verbund.

Die Schlussfolgerung kann deswegen nur wie folgt lauten: Wir können das Votum der Bevölkerung nicht ignorieren und die wichtigsten Neuerungen im Bereich der GASP, die Schaffung des Amtes des Außenministers der Union und den Europäischen Auswärtigen Dienst, so wie sie in der Verfassung vorgesehen sind, umsetzen. Aber wir können und müssen trotzdem auf der Grundlage der gültigen Verträge für mehr Effizienz und Kohärenz der europäischen Außenpolitik Sorge tragen. Das heißt, wir müssen die Instrumente, über die der Rat verfügt - politische, diplomatische Mittel und das zivile und militärische Krisenmanagement - gemeinsam mit den Instrumenten der Kommission - Wirtschafts- und Finanzhilfe, Entwicklungszusammenarbeit, Handelspolitik et cetera - wirksamer und kohärenter zum Einsatz bringen. Das Gleiche gilt für eine stärkere Abstimmung zwischen ,Brüssel ' einerseits und den Mitgliedstaaten andererseits. Es wäre falsch, sich jetzt zurückzuziehen und eine Auszeit zu nehmen. Wir können uns dies angesichts der vielen Herausforderungen, mit denen wir konfrontiert sind, nicht erlauben!

\section{Optionen zur Verbesserung von Effizienz und Kohärenz der GASP}

Was kann unternommen werden, damit wir die genannten Ziele trotz des vorläufigen Scheiterns der Verfassung dennoch erreichen?

- Das Amt des Außenministers der Union ist ohne Vertragsänderung nicht zu realisieren. Dennoch kann der Hohe Repräsentant für die GASP, Javier Solana, der vom Europäischen Rat ja bereits als erster EU-Außenminister designiert worden ist, gestärkt werden. Die jeweiligen Präsidentschaften könnten - um eine stärkere Kontinuität und auch Effizienz zu erreichen - von der Möglichkeit der Aufgabendelegation an den Hohen Repräsentanten verstärkt Gebrauch machen. Zwar kann ihm der Vorsitz im Rat für Außenbeziehungen formell nicht übertragen werden, aber große Teile der Tagesordnung könnten seiner Verhandlungsführung anvertraut werden, insbesondere Themen, bei denen Javier Solana schon heute eine maßgebliche Rolle spielt, wie beispielsweise beim westlichen Balkan, im Nahen Osten oder bei allen Operationen im Rahmen der Europäischen Sicherheits- und Verteidigungspolitik. Schon jetzt hat Javier Solana den Vorsitz des Politischen und Sicherheitspolitischen Komitees inne, wenn dieses sich mit dem NATO-Rat trifft.

- Auch in der Außenvertretung der Union, im politischen Dialog mit Partnern außerhalb der Europäischen Union könnte Javier Solana eine stärkere Rolle übernehmen. Schon heute ist er wichtiger Ansprechpartner amerikanischer Regierungsvertreter, er wurde von Präsident Putin im kleinsten Kreis empfangen, er ist Mitglied des Nahost-Quartetts et cetera. Offizielles Format für den Dialog mit Drittstaaten auf Ministerebene ist bis jetzt die so genannte Troika, die sich zusammensetzt aus den Außenministern der jeweiligen Präsidentschaft und der nachfolgenden Präsidentschaft, dem Hohen Repräsentanten und der Kommission. Die wechselnde Zusammensetzung verhindert Kontinuität, verwirrt die Gesprächspartner und ist zum Krisenmanagement nicht geeignet. Die Übertragung von Dialogverpflichtungen an den Hohen Vertreter - gegebenenfalls gemeinsam mit einem Mitglied der Kommission - würde die dargestellten Nachteile verhindern und für mehr Kontinuität sorgen. Angesammelte Erfahrung könnte besser genutzt, Autorität ausgebaut werden. Kommende Präsidentschaften, insbesondere die deutsche Präsidentschaft im ersten Halbjahr 2007, könnten hier neue Maßstäbe setzen. 
- Die Zusammenarbeit zwischen dem Hohen Repräsentanten und der Kommission könnte verstärkt werden, um Effizienz und Kohärenz der GASP zu erhöhen. Javier Solana und der für Entwicklungspolitik zuständige Kommissar Louis Michel haben in den letzten Monaten zwei gemeinsame Reisen nach Afrika unternommen - einmal in den Kongo, wo die Europäische Union mit verschiedenen Aktionen engagiert ist, und einmal zu einem von der Afrikanischen Union zum Thema ,Sudan“ einberufenen Gebertreffen. In der Balkanpolitik hat sich auch die Zusammenarbeit mit dem Amtsantritt Olli Rehns intensiviert, insbesondere zur wichtigen Frage der Konditionierung unserer Beziehungen. Im Bereich der Nachbarschaftspolitik, dem Zuständigkeitsbereich der Kommissarin Ferrero-Waldner, gibt es ebenfalls ein enormes Potenzial für eine engere Zusammenarbeit, die auch auf der Ebene der Mitarbeiter ausgebaut und systematisiert werden kann. So bin ich beispielsweise mit der Kommission übereingekommen, bei meinen Planungsstabsgesprächen in der Regel Kommissionsvertreter hinzuzunehmen. Weitere Kooperationsmöglichkeiten sind die engere Abstimmung der Kabinette, der Übergang zum Grundsatz gemeinsamer Politikvorschläge, gemeinsame Reisen und ein stärkerer Personalaustausch.

- Auch die über 125 Delegationen der Kommission könnten in diese engere Zusammenarbeit einbezogen werden, ohne dass sie deswegen bereits zu offiziellen EU-Vertretungen werden. So könnten an besonders wichtige Delegationen politische Referenten aus dem Stab Javier Solanas oder den Mitgliedstaaten entsandt werden. Das Aufgabengebiet der Kommissionsdelegationen würde dadurch sinnvoll erweitert, und Javier Solana sowie sein Stab hätten direkte Ansprechpartner unmittelbar vor Ort. Mit der Aufnahme eines politischen Referenten würde sich das Aufgabenspektrum der Delegationen, das sich bisher im Wesentlichen auf Wirtschafts- und Finanzhilfe, Entwicklungs- und Handelspolitik beschränkt, erweitern und zu einer wirksameren Vertretung der Interessen der Union in Drittstaaten führen.

- Zu Beginn hatte ich bereits auf die Notwendigkeit einer stärkeren Kohärenz auch zwischen Brüssel und den Mitgliedstaaten hingewiesen. Dies könnte relativ einfach erreicht werden: Mein eigener Stab, die den Hohen Repräsentanten Javier Solana unterstützende ,Policy Unit", setzt sich gemäß Beschluss des Ausschusses der Ständigen Vertreter zusammen aus je einem Angehörigen der Auswärtigen Dienste der Mitgliedstaaten und der Kommission sowie aus drei Kollegen des Ratssekretariats. Auf Grund dieser Zusammensetzung wurde die ,Policy Unit' gelegentlich schon als ,Embryo“ des Europäischen Auswärtigen Dienstes bezeichnet. So wie ihn die Verfassung vorgesehen hat, kann dieser jetzt nicht kommen, aber es wäre möglich, durch einen einfachen Beschluss der Ständigen Vertreter den zahlenmäßigen Umfang der ,Policy Unit' zu erhöhen und damit die für eine stärkere Kohärenz und Effizienz erforderliche enge Verschränkung zwischen der Arbeit des Hohen Repräsentanten, der Kommission und der Mitgliedstaaten zu erreichen.

\section{Außenpolitische Erfolge lassen sich nur im Verbund erzielen}

Ziel aller dieser kleinen Schritte ist es zu erreichen, was die Bevölkerung laut allen Umfragen will: die Europäische Union in die Lage zu versetzen, erfolgreich auf die Herausforderungen der Globalisierung zu reagieren. Lassen Sie mich wiederholen: Einzelstaaten in der Union - auch Deutschland - werden es allein nicht schaffen, den Balkan langfristig zu stabilisieren oder im Nahen Osten einen wesentlichen Beitrag zu einem erfolgreichen Verlauf des Friedensprozesses zu leisten. Selbst zu Dritt haben Großbritannien, Frankreich und Deutschland keine Chance, Iran zum Einlenken im Nuklearstreit zu bewegen. Es bedurfte der Einbindung der Europäischen Union, um ein Paket zu schnüren, das die Iraner überzeugen 
könnte. Auch wird es im Alleingang nicht gelingen, afrikanische Krisenherde zu stabilisieren, die mittelbar - beispielsweise auf Grund von Flüchtlingsströmen - Auswirkungen auf die Union haben können. Dies gilt auch für Versuche, das Abdriften einzelner Länder zu ,failed states', zu gescheiterten Staaten zu verhindern, die ein Hort für Organisiertes Verbrechen, Drogenhandel und Terrorismus werden können, sowie der Aufgabe, Konflikte in unserer Nachbarschaft beizulegen wie etwa in Weißrussland, Moldawien (Transnistrien) oder im Südkaukasus, die auch direkt oder indirekt negative Folgen für die Union haben.

Trotz dieser Argumente wird von Kritikern oft gefragt, was wir Europäer im Kongo, im Sudan oder in Afghanistan zu schaffen hätten. Neben dem Hinweis auf die konkreten Gefahren für unsere Sicherheit, die auch von geografisch weit entfernten Orten drohen, muss den Kritikern auch etwas Grundsätzliches entgegengehalten werden: Die Europäische Union ist auch und vor allem eine Wertegemeinschaft. Wir können uns nicht damit begnügen, schlimmsten Menschenrechtsverletzungen wie beispielsweise während der Neunzigerjahre auf dem Balkan oder in Burundi/Ruanda tatenlos zuzusehen. Im letzten Jahrzehnt war dies vielleicht noch erklärbar mit dem mangelnden politischen Willen oder den fehlenden Instrumenten, um als Europäer einzuschreiten. Heute trägt dieses Argument nicht mehr. Ob wir dies wollen oder nicht: Als Union, die heute 450 Millionen Menschen umfasst, ein Viertel des Bruttosozialprodukts der Welt erwirtschaftet, größte Handelsmacht und wichtigster Geber ist, müssen wir Verantwortung übernehmen - für unsere Bürger und die in Drittstaaten. Wir sind gefordert, einen Beitrag zu leisten zur Schaffung von Sicherheit und Stabilität, Demokratie und Rechtstaatlichkeit und zur Wahrung von Menschenrechten.

\section{Erfolg durch die Zusammenarbeit mit starken Partnern}

Natürlich können wir das nicht ohne Hilfe schaffen. Wenn wir die genannten Ziele erreichen wollen, müssen wir mit unseren Partnern eng kooperieren: Erstens verdeutlichen die Probleme wie etwa im Nahen Osten oder in Afghanistan, dass wir für Lösungen die Zusammenarbeit mit den USA brauchen. Umgekehrt - das zeigt der Irak - sind auch die USA nicht in der Lage, mit den Herausforderungen allein zurechtzukommen. Zu Beginn seiner zweiten Amtszeit hat Präsident Bush mit seinem Besuch bei der Europäischen Union in Brüssel deutlich gemacht, dass er die Zusammenarbeit mit der Union sucht und stärken will.

Zweitens hat sich die Möglichkeit der Union, zur Bewältigung von Krisen auf die Fähigkeiten der NATO zurückgreifen zu können, bereits bei den EU-Einsätzen in Mazedonien und Bosnien als sehr nützlich erwiesen. Um unnötige Duplizierungen auch in der Zukunft zu verhindern, sollten die Beziehungen zwischen den beiden Organisationen weiter ausgebaut werden. Voraussetzung dafür ist allerdings, dass die NATO bald mit allen EU-Mitgliedstaaten, das heißt also auch mit Zypern und Malta, Sicherheitsabkommen schließt, wodurch ein umfassender Dialog zu allen wichtigen sicherheitspolitischen Themen ermöglicht würde.

Drittens wurde auf dem letzten Gipfeltreffen zwischen der Europäischen Union und Russland am 9. Mai 2005 eine engere Zusammenarbeit in den so genannten vier ,Räumen' vereinbart, unter anderem auch im Bereich der Außen- und Sicherheitspolitik. Zur Bewältigung der verschiedensten Herausforderungen - Verbreitung von Massenvernichtungswaffen, Terrorismus, regionale Krisen, und so weiter - ist eine engere Zusammenarbeit auch mit diesem Partner unabdingbar. Gerade zur Lösung von Problemen in der gemeinsamen Nachbarschaft - Stichwort Moldawien oder Südkaukasus - sollte die Union von den Möglichkeiten, die die Gipfelvereinbarung eröffnet, Gebrauch machen.

Viertens werden in der Europäischen Sicherheitsstrategie neben den USA und Russland auch China, Japan, Indien, Kanada und Brasilien als strategische Partner der Europäischen Union identifiziert. So wie es gilt, diese Partnerschaften weiter zu entwickeln, muss auch die 
Zusammenarbeit mit regionalen Organisationen verstärkt gesucht werden. Insbesondere die Afrikanische Union (im Hinblick auf die Sudankrise) und ASEAN (beispielsweise mit Blick auf eine Lösung der Aceh-Problematik) seien hier genannt.

Und fünftens spielt die UNO eine wichtige Rolle. In ihrem Krisenmanagement bewegt sich die Union nicht nur grundsätzlich im Rahmen der Charta der Vereinten Nationen (VN), es ist ihr auch ein Anliegen, die VN weiter zu stärken. Dies erfolgt nicht nur durch Beiträge zu den laufenden Reformbemühungen, sondern auch durch konkrete Operationen wie im Osten Kongos im Sommer 2003. Auch der Aufbau der EU-Instrumente zum Krisenmanagement (wie etwa die so genannte ,Battle Group') erfolgt mit Blick auf die Möglichkeit, sie gegebenenfalls auch für VN-Operationen zur Verfügung zu stellen.

Der Vergleich zwischen der Europäischen Union und einem Fahrrad ist bekannt: Beide müssen sich fortbewegen sonst stürzen sie um. Nach den fehlgeschlagenen Referenden dürfen wir jetzt nicht zurückfallen in eine Stimmung, wie sie zu Beginn der Achtzigerjahre herrschte, als das Wort von der ,Eurosklerosis‘ die Runde machte. Für den Bereich der Gemeinsamen Außen- und Sicherheitspolitik bestehen für mich keine Zweifel, dass wir auf dem 1999 mit dem In-Kraft-Treten des Vertrags von Amsterdam und dem Amtsantritt Javier Solanas eingeschlagenen Weg fortfahren müssen: Dies erwarten die Bürger in allen EU-Mitgliedstaaten, dies erwarten unsere Partner, und anders sind die Herausforderungen, mit denen sich die Europäische Union heute konfrontiert sieht, nicht zu bewältigen.

Das gesamte Nomos Programm suchen $"$ finden $\rightsquigarrow$ bestellen unter www.nomos.de

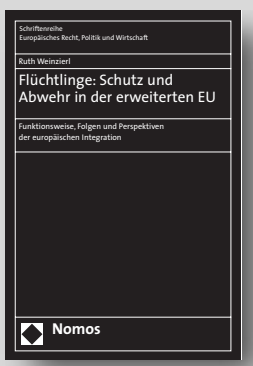

\section{Flüchtlinge: Schutz und Abwehr} in der erweiterten EU

Funktionsweise, Folgen und Perspektiven der europäischen Integration

Von Ruth Weinzierl

2005, 388 S., brosch., 68,- €, ISBN 3-8329-1177-4

(Schriftenreihe Europäisches Recht, Politik und Wirtschaft, Bd. 308)

Das Buch enthält eine umfassende Darstellung und Analyse des EU-Flüchtlingsrechts und der Osterweiterung der EU bis zum 1. Mai 2004. Völkerrecht, Europarecht und nationales Recht werden untersucht. Die Besonderheit des Buches liegt in der gelungenen Verbindung von formeller und inhaltlicher sowie rechtlicher und politischer Analyse.

\section{Nomos}

